\title{
Rendimiento de cultivares de linos oleaginosos en dos series de suelos del noroeste de Corrientes.
}

\author{
A.R. Ferrero, P.A. Garcia y C.N. Balbi \\ Cátedra de Cultivos 1 - Facultad de Ciencias Agrarias. UNNE.
}

\section{Resumen}

Fue analizado el comportamiento varietal de linos oleaginosos, a través de su rendimiento en semillas, en dos suelos de la Provincia de Corrientes, mediante la realización de cuatro ensayos comparativos durante tres campañas, encontrándose diferencias en los rendimientos medios según las características de los suelos comparados.-

En el suelo identificado como perteneciente a la serie Paso Patria, de textura superficial franco-arenosa, en el primer año (1993) se obtuvo una media de $1207 \mathrm{Kg}$ ha $^{-1}$ con el mejor rendimiento para Reconquista INTA con $1231 \mathrm{Kg} \cdot \mathrm{ha}^{-1}$; y en el segundo año (1994), el promedio fue de $1158 \mathrm{Kg} \mathrm{ha}^{-1}$, destacándose Malabrigo INTA con $1198 \mathrm{Kg} \cdot \mathrm{ha}^{-1}$.-

En el suelo perteneciente a la serie Ensenada Grande, textura arenosa, en el primer año (1994) se obtuvo una media de $914 \mathrm{Kg} \cdot$ ha $^{-1}$ con un máximo de $1028.2 \mathrm{Kg} \cdot \mathrm{ha}^{-1}$ para Tape Paraná INTA; y en el segundo año (1995), la media fue de $968.9 \mathrm{Kg} \cdot$ ha $^{-1}$, con un rendimiento máximo de $1087.7 \mathrm{Kg}$ ha $^{-1}$ para Malabrigo INTA.-

Palabras claves: lino, rendimiento, suelos, Corrientes.

\section{Summary}

Oleaginous flax varietal behavoir was analized through seed yield, in two soils from Corrientes Province, with four trials during three years. Mean yield differences were found according to soil characteristics. In the soil identified as Paso Patria Serie, sandy loan surface texture, the first year (1993) a mean yield of $1207 \mathrm{Kg}$. ha ${ }^{1}$ was recorded; Reconquista INTA had the best yield with $1231 \mathrm{Kg} \cdot \mathrm{ha}^{-1}$. During the second year (1994) the mean yield was $1158 \mathrm{Kg} \cdot \mathrm{ha}^{-1}$; Malabrigo INTA produced the maximun yield whit $1198 \mathrm{Kg}$.ha'.- In the soil clasified as Ensenada Grande Serie, with sandy texture, the first year (1994) mean yield was $914 \mathrm{Kg}^{-h^{-1}}$, with a maximun of $1028,2 \mathrm{Kg}^{-h^{-1}}$ for Tape Parana INTA. The second year (1995) mean production was $968,9 \mathrm{Kg} \cdot \mathrm{ha}^{-1}$, with a maximun of $1087,7 \mathrm{Kg} . \mathrm{ha}^{-1}$ for Malabrigo INTA.-

Key words : flax, yield, soil, Corrientes.

\section{Introducción y Antecedentes}

Los cultivares de linos oleaginosos, (Linum usitatissimun, L.), difundidos en el país, son sometidos a ensayos en distintas áreas donde el cultivo es una alternativa real de producción.-

Los antecedentes registrados para la zona norte de la Provincia de Santa Fe, muestran una gran variación interanual en los rendimientos, oscilando entre 1174 y 1908 $\mathrm{Kg} \cdot \mathrm{ha}^{-1}$, siendo de destacar el hecho de que en condiciones de ambiente favorable, con registros de mayores rendimientos, la diferencia entre variedades es escasa, y por el contrario, en condiciones desfavorables, los rendimientos decaen y se destacan algunas variedades.(Corti et al, 1992).

En la misma zona y en tres campañas consecutivas, en chacras de productores miembros de la cooperativa de Avellaneda, se han registrado rendimientos con variaciones entre 1482 y $1614 \mathrm{Kg}$.ha'.'. (Gregoret, 1986).-

En la zona central de Santa $\mathrm{Fe}$, en un ensayo de 9 variedades, se informó de rendimientos promedios de $1391 \mathrm{Kg} \cdot \mathrm{ha}^{-1}$, con valores máximos y mínimos de 1687 y 1142 Kg.ha', respectivamente, que fueron considerados en general buenos para el área.(Fontanetto, 1991); y en otro ensayo, realizado durante la campaña 92/93, se evaluaron 11 variedades con rendimientos que oscilaron entre 1862 y 1104 Kg.ha'. (Fontanetto, 1993).-

En Pergamino, con 12 materiales evaluados, se obtuvieron rendimientos promedios de $1273 \mathrm{Kg} \cdot \mathrm{ha}^{\prime}$, con valores extremos de 1071 y $1422 \mathrm{Kg} \cdot \mathrm{ha}^{-1}$ (Acosta y Marinesco, 1980) y entre 1014 y $1208 \mathrm{Kg}$ ha $^{-1}$ (Acosta y Oliva.1985).;y en 10 ensayos zonales entre 1981 y 1984, los rendimientos oscilaron entre 1089 y $1768 \mathrm{Kg} \cdot \mathrm{ha}^{-1}$ (Acosta et a!. 1984). -

Estos ensayos realizados en distintas áreas agro-ecológicas, han permitido establecer el potencial productivo de cada una de ellas y definir las expectativas de rendimientos de los distintos materiales que se van obteniendo.-

En la provincia de Corrientes, por lo menos en las últimas décadas, no se registra información experimental que cuantifique, ni el potencial de rendimiento de la zona como posible productora, ni el comportamiento de los distintos materiales actualmente disponibles; a pesar de ser la Provincia en su totalidad identificada como formando parte de un área agroecológica apta para su cultivo, con fecha de siembra óptima entre primeros días de mayo y mediados de junio. (Acosta, 1980).-

En el presente trabajo se ha pretendido explorar las posibilidades para el cultivo del lino en Corrientes, y evaluar el comportamiento de distintos cultivares disponibles, a través del rendimiento en semilla, en dos series de suelos en la Provincia.-

\section{Materiales y Métodos}

Durante las campañas agricolas 1993, 94 y 95, se realizaron 4 ensayos de rendimiento con distintos cultivares de lino.- En las dos primeras campañas ( 1994 y 1995) se trabajó en el Departamento San Cosme, en un suelo identificado como Albacualf vértico, arcilloso fino, hipertérmico, perteneciente a la serie Paso Patria,.-

Durante las campañas 1994 y 1995, los ensayos se 
realizaron en el Departamento Capital , en un suelo perteneciente a la serie Ensenada Grande, identificado como Udipsamment alfico, mixto, hipertérmico.( Escobar et al. 1994).-

Con anterioridad a las siembras se extrajeron muestras compuestas de suelos en cada uno de los sitios a dos profundidades ( $0-20$ y $21-40 \mathrm{~cm}$ ), y se determinó en las mismas: $\mathrm{pH}$ (suspensión en agua 1: 2,5 ) ; Materia Orgánica, ( Wackley-Black); Fosforo ( B.Y.K. №1) y Potasio (Extracción por el método de Melich).-

En todos los casos, el diseño fue de bloques completos al azar con parcelas de seis metros cuadrados, utilizando 800 semillas viables por metro cuadrado.

En el primer año (1993), se evaluaron tres cultivares, en siembra realizada el 15 de junio, con 80 días de barbecho.Los materiales ensayados fueron. Malabrigo INTA, Reconquista INTA y Tape INTA.-

En el segundo año, (1994), se repite el ensayo, con 5 cultivares, agregándose a los del año anterior, Salto INTA y Rojas INTA., con siembra realizada el 20 de junio y con un barbecho de 90 dias.-

Los ensayos fueron hechos sobre campo natural, trabajado con arado de disco y rastra. La siembra y el control de malezas fue realizado en forma manual , efectuándose una fertilización de base de $50 \mathrm{~kg}$.ha" de urea y $50 \mathrm{Kg} \cdot \mathrm{ha}^{-1}$ de superfosfato.-

En forma simultánea, el mismo año, en el Departamento Capital se evalúan los 9 cultivares siguientes: Apolo INTA, Tague, Chana, Paisano INTA, Salto INTA, Rojas INTA, Ceibal INTA, Tape INTA y Malabrigo INTA. Repitièndose el ensayo en la campaña siguiente (1995), con la evaluación de siete cultivares, ya que con Tague y Paisano INTA, no se logró la implantación adecuada por fallas en la germinación de la semilla. El antecesor en ambos ensayos fue rastrojo de maíz y se realizó una fertilización con 60 kg.ha' ${ }^{-1}$ de Urea.-

La cosecha fue realizada en forma manual y los resultados evaluados por Análisis de Varianza y test de Tukey.-

\section{Resultados}

En los cuadros № 1 y 2, se pueden observar los datos correspondientes a la caracterización de los suelos donde se realizaron los ensayos, y la información vinculada con las precipitaciones pluviales ocurridas durante los mismos y los promedios anuales.

Cuadro № 1: Valores Analíticos de los Suelos

\begin{tabular}{|l|c|c|c|c|c|}
\hline Serie de Suelo & Prof.cm & $\mathrm{pH}$ & M.O.\% & P ppm & K ppm \\
\hline \multirow{2}{*}{ Paso Patria } & $0-20$ & 5.8 & 2.2 & 8 & 39 \\
& $21-40$ & 5.2 & 1.8 & 6 & 39 \\
\hline \multirow{2}{*}{ Ensenada Grande } & $0-20$ & 6.0 & 0.9 & 22.8 & 142 \\
& $21-40$ & 5.5 & 0.5 & 11.0 & 36 \\
\hline
\end{tabular}

Cuadro № 2: Precipitaciones ( $\mathrm{mm}$ ), promedio anual y total mensual durante las campañas evaluadas. Dpto. Capital

\begin{tabular}{|c|c|c|c|c|c|c|c|c|c|c|c|c|c|}
\hline Año & E & F & M & A & M & J & J & A & S & O & N & D & Prom. \\
\hline $1976 / 85$ & 177 & 156 & 149 & 157 & 127 & 62 & 42 & 55 & 52 & 108 & 174 & 117 & 1381 \\
\hline 93 & 218 & 32 & 177 & 135 & 60 & 56 & 22 & 36 & 59 & 158 & 275 & 137 & 1365 \\
\hline 94 & 210 & 197 & 135 & 67 & 85 & 95 & 9 & 8 & 60 & 138 & 162 & 46 & 1212 \\
\hline 95 & 160 & 208 & 136 & 76 & 105 & 7 & 30 & 70 & 47 & 80 & 76 & 112 & 1107 \\
\hline
\end{tabular}

"FUENTE : Dirección de Economia y Delegaciones Agropecuarias - M.A.G.I.C.-CORFIENTES.-

En los cuadros № 3 y № 4 se reflejan los rendimientos en grano para los años 1993 y 1994 en suelo Paso Patria y para 1994 y 1995 en suelo Ensenada Grande respectivamente.

\section{Cuadro № 3:}

Rendimiento en grano en seis variedades de Lino para las Campañas 1993 y 1994 en suelo Paso Patria. - Kg.ha'

\begin{tabular}{|l|c|c|}
\hline Variedad & Campaña 1993 & Campaña 1994 \\
\hline Tape Paraná INTA & 1197 a & 1085 b \\
\hline Malabrigo INTA & 1193 a & 1198 a \\
\hline Reconquista INTA & 1231 a & 1192 a \\
\hline Salto INTA & - & 1163 a \\
\hline Rojas INTA & - & 1190 a \\
\hline Media & 1207 & 1158 \\
\hline
\end{tabular}

Letras iguales indican que no hay diferencias significativas al $5 \%$ 


\section{Cuadro № 4:}

Rendimiento en grano en ocho variedades de Lino para las Campañas 1994 y 1995 en suelo Ensenada Grande. - Kg.ha'

\begin{tabular}{|c|c|c|c|c|}
\hline Variedad & \multicolumn{2}{|c|}{ Campaña 1994} & \multicolumn{2}{|c|}{ Campaña 1995} \\
\hline ApoloINTA & 923 & a & 937 & bc \\
\hline Tague & 867 & a & - & \\
\hline Chana & 937 & a & 903 & c \\
\hline Paisano INTA & 792 & $\mathbf{a}$ & - & \\
\hline Salto INTA & 1004 & a & 1006 & $a b c$ \\
\hline Rojas INTA & 903 & a & 917 & bc \\
\hline Ceibal INTA & 882 & a & 870 & c \\
\hline Tape Paraná INTA & 1029,2 & a & 1059,5 & $a b$ \\
\hline Malabrigo INTA & 889 & a & 1087,7 & $\mathbf{a}$ \\
\hline Media & 914 & & 968,9 & \\
\hline
\end{tabular}

Letras iguales indican que no hay diferencias significativas al $5 \%$

\section{Discusión}

De los resultados obtenidos se desprende que las diferencias de producción por unidad de superficie, se muestran favorables en los ensayos realizados en el suelo perteneciente a la serie Paso Patria.-

Durante la comparación de los materiales evaluados en el primer año, no se registraron diferencias significativas entre los mismo; hecho que se verificó en el segundo año con significación al $5 \%$ a favor de Malabrigo INTA, Reconquista INTA, Salto INTA y Rojas INTA.-

En el suelo identificado como Ensenada Grande, de textura gruesa, durante la campaña 1994, no se registraron diferencias significativas entre materiales, aunque se dieron los rendimientos mas bajos de los cuatro ensayos y solamente pasaron el umbral de los $1000 \mathrm{Kg}$.ha', Tape Paraná INTA y Salto INTA.-

Durante la campaña 1995, en el mismo suelo, si bien la media se mantuvo baja, se manifestaron diferencias significativas a nivel del $5 \%$ entre los distintos materiales, destacándose Malabrigo INTA.-

\section{Conclusiones}

Los resultados obtenidos indican que el lino podría ser considerado como una alternativa con limitaciones para la producción agricola en la región, y su consideración como posible rubro productivo, debera estar asociado al tipo de suelo disponible y a los precios relativos de los granos de la citada oleaginosa.-

En razón que la comparación de los rendimientos de los cultivares en los dos suelos considerados en el ensayo solo se realizó durante un año, no es posible la generalización, no obstante es dable concluir acerca de desventajas para los materiales establecidos en suelos mas arenosos.-

Los cultivares Malabrigo INTA y Tape Paraná INTA ( 4 años de ensayos) y Salto INTA y Rojas INTA ( 3 años), mostraron rindes promedios entre 1003,3 y 1092,7 $\mathrm{Kg} \cdot \mathrm{ha}^{-1}$, constituyendose en los materiales mas promisorios.-

\section{Bibliografía}

Acosta, P.; 1980 . Lino para semilla y fibra. Fascículo12:2-Segunda Edición. Tomo II- Enciclopedia Argentina de Agricultura y Jardineria- De. ACME- SACI. Bs. As.

Acosta P y O. Marinesco; 1980. Comportamiento de lino oleaginoso en Pergamino. Informe Técnico № 158 . INTAPergamino.

Acosta, P y C. Oliva; 1985. Evaluación de cultivares de linos oleaginosos en Pergamino(Pcia. Bs.As.). Informe Técnico №229. INTA Pergamino.

Acosta P., 1984. Evaluación de linos oleaginosos en Pergamino. INTA Pergamino. Boletin de Divulgación.-

Corti P.A., A.F. Wuthvich y H.S. Vivas. 1992 Evaluación de Variedades de Lino en tres campañas agrícolas en el Noreste de Santa Fe.- INTA - Reconquista.. Información para Extensión. № $41.5 p$ -

Escobar E. H. ; 1994. Mapa de Suelos de los Departamentos Capital, San Cosme e Itati de la Provincia de Corrientes - Fundación Banco Provincia -Corrientes.-

Fontanetto,H.M.;1991.Comportamiento de cultivares de lino en la EEA Rafaela. Informe Técnico №122. INTARafaela.

Fontanetto, H.M.; 1993. Cultivares de lino en diferentes épocas de siembra. Informe técnico № 160 - INTA Rafaela.-

Gregoret,O.; 1986. Informe sobre lino en el Norte de Santa Fe. SEPAR. Reconquista. 